\title{
Die Institutionalisierung politischer Parteien in Südkorea: Konzeptionelle Anmerkungen und empirische Befunde
}

\author{
Von Patrick Köllner, Hamburg ${ }^{1}$
}

\section{Einleitung}

Politischen Parteien kommt in demokratischen Systemen eine zentrale Rolle zu. Von ihnen wird erwartet, dass sie gesellschaftliche Interessen aggregieren und artikulieren, dass sie politisches (Führungs-)Personal rekrutieren, dass sie als Initiator und Mittler im politischen Entscheidungsprozess fungieren und dass sie schließlich im Parlament und darüber hinaus in konstruktiver Weise Konflikte austragen und lösen. Allgemeiner gefasst sollen Parteien zum einen der Repräsentation und Mobilisierung der Bevölkerung und zum anderen der demokratisch legitimierten Herrschaftsausübung dienen. ${ }^{2}$ Trotz aller Kritik und aller Voraussagen über ihren Niedergang hat sich bis heute noch keine effektive Alternative zu Parteien als organisatorischem Bindeglied zwischen Staat und Gesellschaft gefunden, welche die genannten öffentlichen Aufgaben erfüllen kann. ${ }^{3}$ Vor diesem Hintergrund werden stabile und funktionsfähige Parteien sowie institutionalisierte Parteiensysteme als Voraussetzung für die Konsolidierung und den Erhalt demokratischer Systeme angesehen. ${ }^{4}$

Die Republik Korea, allgemein besser als Südkorea bekannt, gehört zu denjenigen Ländern, die im Rahmen der so genannten dritten Demokratisierungswelle die Transition von einem

Der Verfasser dankt Martina Timmermann sowie den Mitgliedern der Forschungsgruppe "Parteien im Spannungsfeld formaler und informeller Politik" des Deutschen Übersee-Instituts für hilfreiche Anmerkungen und Kommentare. Die alleinige Verantwortung des Verfassers für den Inhalt des Artikels bleibt davon unberührt.

Siehe stellvertretend Winfried Steffani, Parteien als soziale Organisationen. Zur politologischen Parteienanalyse, Zeitschrift für Parlamentsfragen 19 (1988), 549-560.

Gute Übersichten der aktuellen Diskussionen über die Rolle und den Wandel politischer Parteien in demokratischen Systemen bieten Klaus von Beyme, Parteien im Wandel, Wiesbaden, 2000 und die Beiträge in dem von Larry Diamond / Richard Gunther herausgegebenen Band Political Parties and Democracies, Baltimore und London, 2001.

Vgl. Robert Dix, Democratization and the Institutionalization of Latin American Political Parties, Comparative Political Studies 24 (1992), 488-511 (489-490); Scott P. Mainwaring, Rethinking Party Systems in the Third Wave of Democratization. The Case of Brazil, Stanford, 1999, 11-15; Petra Bendel / Florian Grotz, Parteiensysteme in jungen Demokratien der Dritten Welt, in: Joachim Betz / Stefan Brüne (Hrsg.), Jahrbuch Dritte Welt 2001, München, 2001, 47-65. 
autoritären zu einem demokratischen politischen System erlebten. ${ }^{5}$ Während sich seit der “demokratischen Öffnung” Südkoreas 1987 einige wesentliche positive Entwicklungen im politischen System des Landes beobachten lassen, ${ }^{6}$ werden die südkoreanischen Parteien in ihrer gegenwärtigen Form weiterhin als Hindernis für die demokratische Konsolidierung des Landes begriffen. Politische Beobachter in Südkorea und anderswo argumentieren, dass die Parteien nicht in der Lage sind, den eingangs erwähnten funktionalen Anforderungen zu entsprechen. Als ein Hauptproblem der südkoreanischen Parteien wird in diesem Zusammenhang deren schwache Institutionalisierung angeführt. ${ }^{7}$

An diesen Befund anknüpfend soll in diesem Artikel zum einen der Frage nachgegangen werden, wie es um die Institutionalisierung südkoreanischer Parteien tatsächlich bestellt ist. Zum anderen soll die Frage beantwortet werden, wie es zu der spezifischen Entwicklung der südkoreanischen Parteien gekommen ist. ${ }^{8}$ Im ersten Schritt der Untersuchung des Zustandes und der Entwicklung der Parteien in dem ostasiatischen Land werden zunächst verschiedene Sichtweisen zu und Konzeptionen von Institutionalisierung diskutiert. Im zweiten Schritt werden Samuel Huntingtons Kriterien der Institutionalisierung auf den Fall südkoreanischer Parteien angewandt. In einem dritten Schritt werden die Ursachen der spezifischen Entwicklung der politischen Parteien in Südkorea thematisiert. Abschließend

Siehe dazu im Einzelnen Aurel Croissant, Politischer Systemwechsel in Südkorea (1985-1997), Hamburg, 1998.

6

So wurde 1993 mit dem ehemaligen Dissidenten Kim Young-sam zum ersten Mal seit über dreiBig Jahren wieder ein Zivilist Staatspräsident. Während seiner Amtszeit wurde nicht nur das Militär des Landes vollständig von der politischen Bühne verbannt, sondern auch die lokale Autonomie formal wiederhergestellt. Bereits achtmal fanden zudem zwischen 1987 und 2002 freie und faire, wenn auch mit hohem Finanzaufwand betriebene Wahlen für die Nationalversammlung und das oberste Regierungsamt statt. Bei den Präsidentschaftswahlen 1997 konnte sich dabei erstmals ein Kandidat der Opposition, der Politiker-Veteran Kim Dae-jung, durchsetzen. Gute Überblicke über die verschiedenen Aspekten der demokratischen Konsolidierung Südkoreas bieten die Bände von Larry Diamond / Byung-Kook Kim (Hrsg.), Consolidating Democracy in South Korea, Boulder / London, 2000 sowie Larry Diamond / Doh Chull Shin (Hrsg.), Institutional Reform and Democratic Consolidation in Korea, Stanford, 2000.

Siehe etwa Hyug Baeg Im, South Korean Democratic Consolidation in Comparative Perspective, in: Diamond / Kim (Hrsg.), a.a.O., 21-52 (32-34); Hoon Jaung, Reform of Political Parties: From 'Cadre' to National Interests, Korea Focus 7 (1999), 57-69; ders., Electoral Politics and Political Parties, in: Diamond / Shin (Hrsg.), Institutional Reform 2000, 43-71; Yong-Ho Kim, Grassroots Parties Essential for Political Reform, Korea Focus 10 (2002), 17-22.

Ausgeblendet bleibt hier mithin die Frage nach den Auswirkungen des Zustands der Parteien auf den Prozess der demokratischen Konsolidierung in Südkorea. Dieser Frage gehen an anderer Stelle aus unterschiedlicher Perspektive Croissant (Aurel Croissant, Demokratische Entwicklung in den Philippinen, Südkorea und Thailand. Von der Transition zur defekten Demokratie, unveröffentlichte Dissertation, Universität Mainz, 2001, 290-293) und Köllner (Patrick Köllner, Die gesellschaftliche Verankerung politischer Parteien in Südkorea, Arbeitspapier, Deutsches Übersee-Institut, April 2002, 20-23) nach. 
werden die Ergebnisse der Untersuchung sowie deren konzeptionelle Implikationen zusammenfassend diskutiert.

\section{Die Institutionalisierung politischer Parteien: Sichtweisen und Konzeptionen}

Die wissenschaftliche Auseinandersetzung mit dem Thema der Entwicklung politischer Parteien zieht sich, angefangen mit klassischen Studien wie der von Robert Michels, ${ }^{9}$ wie ein roter Faden durch die Parteienforschung der letzten rund hundert Jahre. Wie jedoch die Stabilisierung von Parteien (als Organisationen und bei Wahlen) oder die Routinisierung von Regeln, Prozeduren und Verhalten innerhalb der Parteien konzeptionell erfasst und anhand von Indikatoren beurteilt werden kann, blieb dabei zunächst weitgehend im Dunkeln.

Konzeptionelle Ansätze zur Beschäftigung mit diesen Aspekten der Entwicklung politischer Parteien wurden schließlich aus der soziologischen Organisationsforschung und der Forschung über politische Entwicklung bzw. "Modernisierung" entliehen. So hatte sich bereits ab den späten 40er Jahren Philip Selznick ${ }^{10}$ mit dem Thema der Institutionalisierung von Organisationen und Prozessen beschäftigt. Selznick und sein Kollege Broom definierten Institutionalisierung als "die Herausbildung geordneter, stabiler, gesellschaftlich integrierender Muster aus instabilen, lose organisierten oder eng gefassten technischen Aktivitäten". 11 Bereits einige Jahre zuvor hatte Selznick ausgeführt, das Institutionalisierung etwas ist

,that happens to an organization over time, reflecting the organization's own distinct history, the people who have been in it, the groups it embodies and the vested interests they have created, and the way it has adapted to its environment. In what is perhaps its most significant meaning, to institutionalise is to infuse with value beyond the technical requirements of the task at hand." 12

Während Selznick andere Aspekte von Institutionalisierungsprozessen wie die Schaffung einer formalen Struktur, die Entwicklung informeller Normen, die selektive Rekrutierung von Organisationsmitgliedern oder administrative Rituale und Ideologien, die aus der

Man denke an seine bekannte These vom "ehernen Gesetz der Oligarchie" (Michels 1989 [1911]: 351-369).

10 Philip Selznick, Foundations of the Theory of Organization, American Sociological Review 13 (1948), 25-35; ders., Leadership in Administration: A Sociological Interpretation, New York, 1957.

11 Leonard Broom / Philip Selznick, Sociology: A Text with Adapted Readings, New York, 1955, 238, Übersetzung P.K.

12

Selznick (1948, Fn. 10), zitiert nach Sigmund Akselsen / Bente Evjemo / Bente L. Kassah / Pål S. Malm / Marianne Moe / Beryl Nordhus / Pål Ytterstad, Network Organizations and Information Systems: Challenges and Relevant Theories, 1997, unveröffentlichtes Manuskript, 8. 
spezifischen Geschichte einer Organisation resultieren, keineswegs übersah, ${ }^{13}$ war für ihn der Aspekt der "Wertinfusion" am Bedeutendsten. Von Selznick wurde eine Organisation dann als institutionalisiert angesehen, wenn ihre Mitglieder sie nicht (länger) als reines Instrument zur Erreichung bestimmter Ziele begreifen, sondern ihr einen darüber hinaus gehenden "Wert" zumessen. Mithin müssen die Mitglieder auch unabhängig von der Erreichung der relevanten Ziele am Erhalt der betreffenden Organisation bzw. des betreffenden Prozesses interessiert sein. "Wertinfusion" meint in diesem Zusammenhang also "Wertschätzung", 14 die ihren Ausdruck in einem langfristigen "Commitment" der Organisationsmitglieder gegenüber der Organisation bzw. dem fraglichen Prozess findet. Der entscheidende Test für Institutionalisierung in diesem Sinne bestand für Selznick in ihrer "Entbehrlichkeit" (expendability), d.h. "the readiness with which the organization or practice is given up or changed in response to new circumstances or demands". 15

Samuel Huntington griff Selznicks Sichtweise von Institutionalisierung in seinem einflussreichen Werk „Political Order in Changing Societies“ auf und definierte Institutionalisierung als den Prozess, durch den Organisationen und Prozeduren Wert und Stabilität erlangen. Nach Huntington ${ }^{16}$ bezeichnet ein hoher Grad an Institutionalisierung eine große Anpassungsfähigkeit, Komplexität, Autonomie und Kohärenz dieser Organisationen und Prozeduren (siehe auch unten). Interessanterweise beziehen sich Huntingtons Kriterien auf verschiedene Attribute der relevanten Organisationen und Prozesse als solcher, während der Aspekt der Wertschätzung durch Organisationsmitglieder von ihm nicht weiter vertieft wurde.

Auch Kenneth Janda, der sich 1980 mit der Institutionalisierung von Parteien - also nicht mehr Organisationen im Allgemeinen - auseinander setzte, führte in diesem Kontext zunächst grundlegende Aspekte der Stabilität, der Routinisierung und der Wertschätzung an, um in letzter Instanz doch nur den erstgenannten Aspekt zu vertiefen. Janda ${ }^{17}$ definierte eine institutionalisierte Partei als "one that is reified in the public mind so that "the party" exists as a social organisation apart from its momentary leaders, and this organisation demonstrates recurring patterns of behaviour valued by those identified with it". Von zentraler definitorischer Bedeutung waren für Janda also einerseits die gesellschaftliche Wahrnehmung der Parteien als eigenständige Organisationen (deren Existenz nicht an einzelne Führungspersonen gebunden ist) und andererseits "verregelmäßigte" und

Vgl. auch Philip Selznick, Institutionalism 'old' and 'new', Administrative Science Journal 41 (1996), 270-278.

14 In den Worten von Selznick (1957, Fn. 10), 17 bezeichnet Wert "the pricing of the [organization] for its own sake".

15 Selznick (1996, Fn. 13), 2.

16 Samuel P. Huntington, Political Order in Changing Societies, New Haven, 1968, 12.

17

Kenneth Janda, Political Parties. A Cross-National Survey, New York / London, 1980, 19. 
geschätzte Verhaltensmuster innerhalb dieser Organisationen. Betrachtet man jedoch die von Janda für die vergleichende Institutionalisierungsanalyse gewählten Indikatoren - das Alter von Parteien, das Ausmaß von Namensveränderungen und organisatorischer Kontinuität, die Legitimität von Parteiführungen und deren Wechsel sowie schließlich die Stabilität von Parteien bei Wahlen und im Parlament ${ }^{18}$ - steht der Stabilitätsaspekt klar im Vordergrund. ${ }^{19}$

Während bei Huntington und Janda eine deutliche Diskrepanz zwischen der definitorischen und der operationalen Annäherung an die Institutionalisierung von Organisationen bzw. Parteien besteht, hat Steven Levitsky in den vergangenen Jahren für eine bewusste konzeptionelle Trennung der Aspekte der Wertschätzung einerseits und der Stabilität sowie Routinisierung andererseits plädiert. So machte er zunächst am Fall der peronistischen Partei Argentiniens deutlich, dass die hohe Wertschätzung einer Partei (durch die Parteimitglieder und/oder die Öffentlichkeit) nicht einhergehen muss mit einer hohen Stabilität der Organisationsstrukturen und der Routinisierung der Verhaltensmuster in dieser Partei. ${ }^{20}$ Anders ausgedrückt: Die Wertschätzung für eine Partei kann sich auf ähnlich hohem oder niedrigen Niveau bewegen wie deren Stabilität und Routinisierung, muss es aber nicht. Zudem können die verschiedenen Aspekte auch gegensätzliche Auswirkungen, etwa auf die organisatorische Anpassungsfähigkeit einer Partei, haben. Während eine hohe Wertschätzung für eine Partei deren Anpassungsfähigkeit tendenziell erhöht, kann demgegenüber einer hoher Grad an Routinisierung in der Partei deren Anpassungsfähigkeit verringern. ${ }^{21}$

18

19

20

Janda (Fn. 17), 19-27.

Viele Parteienforscher haben sich in der Folge an den selben oder ähnlichen Indikatoren orientiert und dabei versucht, diese noch stärker zu konkretisieren. So postulierten etwa Richard Rose / Thomas T. Mackie, Do Parties Persist or Fail? The Big Trade-Off Facing Organizations, in: Kay Lawson / Peter H. Merkl (Hrsg.), When Parties Fail. Emerging Alternative Organizations, Princeton, 1988, 533-558 (536), dass eine Partei nach dem Antritt bei drei nationalen Wahlen als institutionalisiert angesehen werden kann. Für umfassendere konzeptionelle Diskussionen der Institutionalisierung politischer Parteien siehe auch Angelo Panebianco, Political Parties: Organization and Power, Cambridge, 1988, Kapitel 3, Robert Harmel / Lars Svåsand, Party Leadership and Party Institutionalization, West European Politics 16 (1993), 67-88 (74-75), Moshe Maor, Political Parties and Party Systems. Comparative Approaches and the British Experience, London / New York, 1997, 66-76 sowie Vicky Randall / Lars Svåsand, Party Institutionalization in New Democracies, Party Politics 8 (2002), 5-29.

Vgl. Steven Levitsky, Institutionalization and Peronism, Party Politics 4 (1998), 77-92. Denkbar ist im Übrigen auch der umgekehrte Fall. Levitsky, 77-92 (88) führt hier das Beispiel einiger ökologischer Parteien in Westeuropa an, in denen organisatorische Fragen - man denke an Quotenregelungen, das Rotationsprinzip und die Trennung von Amt und Mandat - als wichtig erachtet werden, andererseits aber ideologische Fragen von größerer Bedeutung als die Identifikation mit der Partei als solcher sein können.

Siehe auch Steven Levitsky, Transforming Labor-Based Parties in Latin America: The Argentine Justicialista Party in Comparative Perspective, Working Paper \#288, Kellogg Institute of International Studies, University of Notre Dame, 2001, 7-10. 
Angesichts dieser Ambivalenz ist es sinnvoll, Institutionalisierung in seine verschiedenen Aspekte zu unterteilen. In der folgenden Analyse der Institutionalisierung südkoreanischer Parteien sollen dabei zunächst die Aspekte der Stabilität und der Routinisierung untersucht werden, für die ausreichendes empirisches Material zur Verfügung steht. In der in Abschnitt 4 folgenden Diskussion der Ursachen der spezifischen Parteienentwicklung in Südkorea wird dann deutlich werden, dass zumindest die Wertschätzung von Parteien durch politische Führer durchaus deren Institutionalisierung im Sinne der Stabilisierung und Routinisierung beeinflussen kann und damit in analytischer Hinsicht eine unabhängige Variable darstellt.

Beschränkt man die Analyse der Institutionalisierung politischer Parteien auf die Aspekte der Stabilität und der Routinisierung kann man sich denn auch an den bereits genannten Kriterien von Huntington orientieren, die von Dix (1992) in operationaler Hinsicht verfeinert worden sind. So können sowohl das chronologische, d.h. das absolute Alter als auch das "generationale Alter" 22 von Parteien als Indikatoren für ihre Anpassungsfähigkeit an Herausforderungen aus der Umwelt benutzt werden. Ein weiterer Indikator in diesem Zusammenhang ist der erfolgreich überstandene Übergang einer Partei von der Opposition in die Regierung und umgekehrt. Hinsichtlich der Komplexität politischer Parteien gilt das Interesse der funktionalen und organisatorischen Ausdifferenzierung von Parteien bzw. umgekehrt dem Ausmaß des Personalismus in Parteien. Die Autonomie politischer Parteien betrifft das Ausmaß ihrer Unabhängigkeit von anderen sozialen Gruppen (Familie, Clan, Klassen, Elite- und Interessengruppen etc.). Kohärenz schließlich bezieht sich auf das Ausmaß, in dem eine Partei - etwa in Bezug auf ihre Funktionen und Problemlösungsmechanismen - als einheitlicher Akteur agiert. ${ }^{23}$

Das generationale Alter einer Partei lässt sich messen an den Generationswechseln in der Führung einer Partei. Entsprechende Wechsel bezeugen die Fähigkeit einer Partei, unvermeidliche Konflikte zwischen den Generationen zu überstehen; Dix (1992, Fn. 4), 491. chenden Parteiensystems zu messen. Da es uns im Folgenden um die Institutionalisierung südkoreanischer Parteien und nicht des Parteiensystems geht, wird dieser Ansatz hier nicht weiter verfolgt. 


\section{Die Institutionalisierung südkoreanischer Parteien}

\subsection{Anpassungsfähigkeit}

Hinsichtlich des Kriteriums der Anpassungsfähigkeit lässt sich für den südkoreanischen Fall zunächst festhalten, dass die dortigen politischen Parteien relativ kurze Lebenszeiten aufweisen. ${ }^{24}$ Nach Berechnungen von Pae ${ }^{25}$ bestanden zwischen 1947 und Mitte der 80er Jahre über 80 Prozent aller Parteien weniger als vier Jahre. Einzig die langjährigen Regierungsparteien, Syngman Rhees Liberal Party (1951-1960) und Park Chung-hees Democratic Republican Party (1963-1980), sowie die wichtigsten Oppositionsparteien dieser Zeit, die Democratic Party (1954-1965) und die New Democratic Party (1967-1980), wiesen eine mehr als zehnjährige Parteigeschichte auf.

Auch nach der demokratischen Transition hat sich die Lebensdauer der politischen Parteien nicht verlängert; im Gegenteil: Nach Berechnungen von Croissant ${ }^{26}$ lag die durchschnittliche Lebensdauer der zwischen 1987 und 2000 existierenden Parteien infolge von Fusionen, Schließungen und Neugründungen bei nur rund 21/2 Jahren. Keine politische Partei hat seit den ersten freien Wahlen 1998 mehr als zweimal an Parlamentswahlen in Südkorea teilgenommen. Bei den Parlamentswahlen 1992 beispielsweise wies die älteste Partei mit einem Stimmenanteil von über 10 Prozent gerade einmal eine Lebensdauer von zwei Jahren auf! Bemerkenswert in Bezug auf das generationale Alter der politischen Parteien in Südkorea ist zudem, dass mit einer Ausnahme ${ }^{27}$ bisher keine Partei den Tod ihres Parteigründers oder dessen Abgang von der politischen Bühne überlebt hat.

Als weiterer Indikator für die Anpassungsfähigkeit einer Partei kann deren Übergang aus der Rolle der Opposition in die der Regierung angesehen werden. In Südkorea kam es hierzu erst zweimal: Der erste entsprechende Übergang erfolgte 1990 im Rahmen der Fusion der damals oppositionellen Reunification Democratic Party von Kim Young-sam mit der regierenden Democratic Justice Party von Roh Tae-woo und der New Democratic Republican Party von Kim Jong-pil. ${ }^{\mathbf{2 8}}$ Der zweite Übergang erfolgte als Resultat des Sieges von Kim Dae-jung bei den Präsidentschaftswahlen 1997; der von Kim geführte National Congress for New Politics (heute: Millennium Democratic Party) wurde zur Regierungs-

Die folgenden Ausführungen basieren teilweise auf Köllner (2002, Fn. 8), 6, 16-19. Pae (1986, Fn. 54), 155.

Aurel Croissant (2001a), Das südkoreanische Wahlsystem und seine Reform, ASIEN (2001), Nr. 78, 70-90 (78-79).

Vor den Präsidentschaftswahlen 1987 übernahm Roh Tae-woo die Führung der Democratic Justice Party von Chun Doo-hwan.

Kim Young-sam trat 1992 für die aus der Fusion hervorgegangene Democratic Liberal Party bei den Präsidentschaftswahlen an und konnte diese auch gewinnen. 
partei, ohne dabei jedoch über eine eigene Mehrheit in der Nationalversammlung zu verfügen. $^{29}$

\subsection{Komplexität}

Das Kriterium der Komplexität politischer Parteien bezieht sich wie erwähnt zunächst auf deren innerorganisatorische Differenzierung. Zumindest nach eigner Darstellung verfügen die größeren Parteien in Südkorea über ausdifferenzierte Organisationsstrukturen vertikaler und horizontaler (funktionsbezogener) Art. So stellt etwa die vormalige Regierungspartei GNP in ihrer Parteibroschüre ihre Organisation wie folgt dar:

„The Grand National Party (Hannara Party) consists of the Central Party (Party Hq), 16 City \& Provincial Chapters and 253 district parties each of which has a secretariat. [...] The city \& provincial convention consists of 150 to 300 representatives. It performs such functions as electing representatives for the National convention, operating the committees of the Central Committee, choosing the chairmen of city \& provincial chapters, deliberating on matters recommended by the district party, and submitting recommendations of every kind to Central Party. [...] The district convention consists of 100 to 150 representatives. It performs such functions as choosing the chairmen of district chapters, electing representatives for the National Convention, and submitting recommendations of every kind to Central Party. “ 30

In der Realität sind jedoch viele lokale Parteiorganisationen wenig mehr als der persönliche Apparat des jeweiligen Mandatsträgers oder Parlamentskandidaten, der von der Parteizentrale als Ortgruppenleiter im jeweiligen Wahlkreis eingesetzt wird. Er sucht sich seine Mitarbeiter selbst aus, wobei primäre soziale Bindungen (Familie, Verwandte etc.) und informelle Netzwerke oftmals eine zentrale Rolle spielen. ${ }^{31}$ Wichtiger als die formalen Parteistrukturen auf der lokalen Ebene sind für viele Politiker ihre persönlichen Unterstützergruppen (sajojik), die zur Sammlung von Finanzmitteln, zur Stimmenmobilisierung und

Eine Parlamentsmehrheit wurde in der Folge - zumindest zeitweise - über eine Regierungskoalition mit den United Liberal Democrats von Kim Jong-pil sicher gestellt.

GNP (Grand National Party), The Grand National Party, Parteibroschüre, o.J., vermutlich 1999, 7. $\mathrm{Zu}$ den formalen Parteistrukturen der vier größeren südkoreanischen Parteien Mitte der 90er Jahre siehe Yong-Ho Kim (1998), Korea, in: Wolfgang Sachsenröder / Ulrike E. Frings (Hrsg.), Political Party Systems and Democratic Development in East and Southeast Asia. Volume II: East Asia, Aldershot u.a., 132-175 (146-150).

Wonkyoo Lee, Political Culture in the Republic of Korea and Its Effects on Parties and Elections, unveröffentlichte Dissertation, University of Missouri-Columbia, 1995, Kapitel 14, 134-135. 
zur Pflege persönlicher Beziehungsnetzwerke vor Ort dienen. ${ }^{32}$ Wonkyoo Lee ${ }^{33}$ notiert zu der personengebundenen Parteienorganisation auf der lokalen Ebene Folgendes:

„Each district chapter chair develops his own organizational strength by recruiting cadres of workers and soliciting campaign funds himself based on kinship, region or school. [...] The local party organization is just the District Chapter Chairman's personal support organization. [...] Another indication that the Korean party infrastructure is organized privately is that the list of party members is held by the district chapter chair privately, not by the district chapter officially. [...] Centralization of power at the national level and absence of local autonomy go hand in hand with weak grass-roots party organization. Weak grass-roots party organization, low membership levels, and private personal organizations on the local level are closely related to the lack of continuity in party organization and activities. Like American local party organizations, the Korean local opposition party's candidate's headquarters are located in his business office or in his attorney's office. The office is often operated by one man in the local district chapter and performs few functions. “34

Während also die formalen Parteigliederungen auf der lokalen Ebene wenig mehr als eine Hülle für die Aktivitäten einzelner Politiker darstellen, fungieren persönliche Netzwerke als Instrument zur gesellschaftlichen Anbindung. Allerdings weisen die persönlichen Unterstützerorganisationen im südkoreanischen Fall nicht denselben Grad der Institutionalisierung auf wie im benachbarten Japan, wo ihnen teilweise sogar zugesprochen wird, ein funktionales Äquivalent für formale Parteigliederungen auf der lokalen Ebene darzustellen. 35

\subsubsection{Personalismus in südkoreanischen Parteien}

Ein zweiter Indikator für die Komplexität politischer Parteien ist das Ausmaß des Personalismus, wobei hier davon ausgegangen wird, dass eine starke personelle Prägung gleichbedeutend mit einem niedrigen Grad der Institutionalisierung ist. In Anlehnung an Ansell und

Siehe dazu Soohyun Chon, The Election Process and Informal Politics in South Korea, in: Lowell Dittmer / Haruhiro Fukui / Peter N. S. Lee (Hrsg.), Informal Politics in East Asia, Cambridge u.a., 1999, 66-81.

Lee (1995, Fn. 31), 143, 144, 148.

34

Zahlreiche Vorschläge zur Reform der regionalen und lokalen Gliederungen der Parteien liefert eine jüngere Aufsatzsammlung des Zentralen Wahlaufsichtskomitees Südkoreas (Chungang Sôngôkwalli Wiwônhoe, Chôbiyong kohyoyulûi chigudang unyôngpangan [Managementmaßnahmen lokaler Parteiorganisationen zur Kostenreduzierung und Effektivitätssteigerung], Seoul, 1998). 
Fish $^{36}$ kann Personalismus im weitesten Sinne als "Loyalität gegenüber Personen statt gegenüber unpersönlichen Ideologien, Institutionen und Regeln" verstanden werden. Dabei muss Personalismus nicht unbedingt auf dem persönlichem Charisma des jeweiligen politischen Führers beruhen, sondern kann auch auf seinen distributiven Fähigkeiten (Versorgungspatronage), seiner Rolle als Repräsentant eines bestimmten Programms oder einer bestimmten Ideologie oder aber seiner Rolle als Makler und Konfliktmanager innerhalb der Partei fußen. ${ }^{37}$

Südkoreanische Parteien sind stark an einzelnen Führungspersönlichkeiten und deren Netzwerken orientiert (siehe auch oben). Dieses Phänomen wird oftmals auf die verbreitete Orientierung der koreanischen Gesellschaft an Personen statt an abstrakten Prinzipien und Institutionen zurückgeführt. So betont beispielsweise Yong-Ho $\mathrm{Kim}^{38}$, dass

,a dominant feature of South Korean political culture is the strong focus on the person and the authority s/he represents, rather than the institution. Among Koreans there is a tendency to attribute power and authority to individual leaders and not the institutions in which those leaders serve. They seem to feel more comfortable with the idea of personalized authority rather than with the idea of institutionalized power."

In der Tat kann argumentiert werden, diese Orientierung die deutliche Tendenz der Wählerschaft, sich auf Personen statt auf Institutionen und Inhalte zu konzentrieren, unterfüttert hat. ${ }^{39}$ Zumindest gab es nur wenige Faktoren, die dieser personenbezogenen Orientierung entgegenwirkten.

Dies klärt jedoch noch nicht, auf welcher Grundlage der anhaltende Personalismus als Modus der Verbindung zwischen Wählern und Politikern im gegenwärtigen Korea steht. In diesem Zusammenhang kann zunächst festgestellt werden, dass auch das Charisma einzelner politischer Führer - man denke an die ersten Phasen der Regierungen von Syngman Rhee (1948-1960) und Park Chung-hee (1963-1979) - oder von Politikern auf der lokalen Ebene eine Rolle für deren Unterstützung bei den Wählern spielt bzw. gespielt hat. Insgesamt betrachtet kommt aber dem distributionsorientierten Personalismus eine größere Bedeutung zu, d.h. die persönliche Bindung zwischen Wählern und Mandatsträgern hängt eher davon ab, inwieweit der einzelne Politiker in der Lage ist, seinen Wählern individuelle Dienstleistungen sowie materielle Wohltaten individueller und kollektiver Natur (z.B.

Christopher K. Ansell / M. Steven Fish, The Art of Being Indispensable. Noncharismatic Personalism in Contemporary Political Parties, Comparative Political Studies 32 (1999), 283-312, 286.

Siehe Ansell / Fish, ebd., 288-289.

38

Yong-Ho Kim (1998, Fn. 30), 163.

39

Zur Bedeutung der Person des Kandidaten bei der Stimmentscheidung südkoreanischer Wähler siehe etwa Chulsu Kim, Parties and Factions in Korean Politics, unveröffentlichte Dissertation,

40 University of Massachusetts, 1973, 223-224 und Lee (1995, Fn. 31), Kapitel 14.

Siehe auch Lee (1995, Fn. 31), 308-309. 
Infrastrukturprojekte) zukommen zu lassen. Dies gilt insbesondere für ländliche Wahlbezirke. $^{41}$ Diese Art der persönlichen Anbindung stellt für die betreffenden Politiker - nicht zuletzt vor dem Hintergrund des personenzentrierten Wahlsystems in Südkorea ${ }^{42}$ - eine rationale Strategie zur Sicherung der Wiederwahl dar, wie sie auch in anderen Ländern anzufinden ist. ${ }^{43}$

\subsubsection{Regionalismus}

Eine Besonderheit des südkoreanischen Wahlverhaltens ist der starke Regionalismus, der seit der demokratischen Öffnung und dem damit verbundenen Wegfall der alten Konfliktlinie Demokratisierung vs. Status quo deutlich an Bedeutung gewonnen hat. So weisen alle größeren Parteien Südkoreas ausgeprägte regionale Hochburgen auf. ${ }^{44}$ Wesentlich ist hier jedoch, dass innerhalb dieser Hochburgen patronageorientierte Stimmenmobilisierungsstrategien eine wichtige Rolle spielen. Insgesamt lässt sich festhalten, dass südkoreanische Parteien ein überaus starkes Maß an Personalismus aufweisen, der durch lokale Patronageund andere Netzwerke sowie regionale Loyalitäten gestützt wird.

\subsection{Autonomie}

Die Autonomie politischer Parteien lässt sich nach Huntington ${ }^{45}$ an dem Ausmaß ablesen, in dem Parteien unabhängig von anderen gesellschaftlichen Gruppen existieren. ${ }^{46}$ Hinsichtlich des südkoreanischen Falls kann seit der demokratischen Transition des Landes Ende

An dieser Stelle kann nicht näher auf die parochiale Art der Wahlkreisbetreuung in Südkorea eingegangen werden. Siehe hierzu Patrick Köllner, Politische Repräsentation in Südkorea: Rahmenbedingungen, Praxis und die Haltung der Bevölkerung, in: ders. / Rüdiger Frank, Politik und Wirtschaft in Südkorea, Hamburg, 1999, 55-87 (70-74) und die dort zitierte Literatur. Bis Anfang der 90er Jahre spielte für die Regierungspartei auch die Instrumentalisierung der Bürokratie auf zentraler und lokaler Ebene eine zentrale Rolle für Stimmenmobilisierung. Siehe hierzu Chon (1999, Fn. 32), 73-76.

42 Siehe dazu Croissant (2001, Fn. 26); Jongryn Mo / David Brady, The SNTV and the Politics of Electoral Systems in Korea, in: Grofman, Bernard, Sung-Chull Lee, Edwin A. Winckler / Brian Woodall (Hrsg.), Elections in Japan. Korea, and Taiwan under the Single Non-Transferable Vote: The Comparative Study of an Embedded Institution, Ann Arbor, 2001, 211-227.

$\mathrm{Zu}$ den Nutzen und Kosten patronagebasierter Stimmenmobilisierungsstrategien siehe Carolyn M. Warner, Political Parties and the Opportunity Costs of Patronage, Party Politics 3 (1997), 533548.

44 
der 80er Jahre eine Abnahme der Verbindungen zwischen den Parteien und anderen gesellschaftlichen Gruppen ausgemacht werden, die allerdings durchaus auch kritisch betrachtet werden kann. So bestehen im Gegensatz zu den 60er bis 80er Jahren keine engen korporatistischen Beziehungen mehr zwischen der Regierungspartei einerseits und dem Militär, den Unternehmensverbänden und den landwirtschaftlichen Kooperativverbänden andererseits. ${ }^{47}$ Kollektive Mitgliedschaften an Parteien, durch organisatorische Verschränkung o.ä., existieren nicht. Allerdings muss erwähnt werden, dass Spenden von Unternehmensseite angesichts einer mangelnden umfassenden Parteienfinanzierung einerseits und der kostenträchtigen Natur von Wahlkämpfen andererseits für Politiker und Parteien von größerer Bedeutung sind. Fraglich ist dabei, ob derartige Spenden die Autonomie der Parteien einschränken. So sind in der Vergangenheit derartige Spenden des Öfteren als "QuasiSteuern" betrachtet worden, denen keine konkreten Gegenleistungen gegenüberstanden. Allerdings scheint in den 90er Jahren das Ausmaß "echter" Korruption, d.h. Spenden für konkrete Gegenleistungen, zugenommen zu haben. Die damit verbundene Einschränkung von Autonomie bezieht sich aber weniger auf die Parteien per se, sondern eher auf politische Entscheidungsträger in der Regierung. ${ }^{48}$

Beschränkt ist in jedem Fall die Abhängigkeit südkoreanischer Parteien von gesellschaftlichen Gruppen, die anderswo eine wichtige Rolle in der Politik spielen. Als zentrales Beispiel seien hier zunächst die Gewerkschaften genannt. Zwar können diese seit jüngster Zeit auch legal in der Politik aktiv werden, doch bisher ist ihr Einfluss beschränkt, wie unter anderem das schwache Abschneiden der Anfang 2000 gegründeten Democratic Labor Party bei den Parlamentswahlen im selben Jahr signalisiert. Es lässt sich in diesem Zusammenhang allgemein anmerken, dass die südkoreanische Arbeiterschaft, genauso wie die Studentenbewegung, zwar eng mit dem Demokratisierungsprozess verbunden, aber andererseits nicht in der Lage gewesen ist, bedeutsamen Einfluss auf Entscheidungen und Strukturen der Parteien des Landes zu nehmen. Auch existieren zwischen den in den letzten Jahren sprunghaft gewachsenen Nichtregierungsorganisationen (NROs) wie der People's Solidarity for Participatory Democracy oder der Citizens' Coalition for Economic Justice und einzelnen Parteien keine starken Verbindungen; vielmehr verstehen sich diese Organisationen eher als Alternative zur etablierten Parteienpolitik. ${ }^{49}$

Siehe hierzu Chulsu Kim (1973, Fn. 39), Kapitel 4.

Zur Finanzierung politischer Parteien in Südkorea und zum Problem der Korruption siehe Byeong-Seog Park, Political Corruption in South Korea: Concentrating on the Dynamics of Party Politics, Asian Perspective 19 (1995), 163-193 und David C. Kang, Money Politics and the Developmental State in Korea, International Organization 56 (2002), 177-207. Zur Bedeutung der staatlichen Parteienfinanzierung siehe Jin-Young Kwak, Open System or Cartelized System? Redefining the Korean Party System after Democratization, in: Young Rae Kim, Hochul Lee / InSub Mah (Hrsg.), Redefining Korean Politics, Seoul, 2002, 403-448.

Im Vorfeld der Parlamentswahlen 2000 machten südkoreanische NROs durch ihre Internetgestützten Kampagnen gegen "ungeeignete" Kandidaten von sich reden. Siehe dazu Roland Wein, 
Insgesamt lässt sich feststellen, dass die Abhängigkeit der politischen Parteien in Südkorea von externen Gruppen mit Ausnahme der erwähnten Abhängigkeit von Unternehmensspenden begrenzt ist. Es wäre jedoch wenig differenziert, dies nur als Zeichen einer starken Institutionalisierung der Parteien zu sehen. Vielmehr kann argumentiert werden, dass die mangelnde Vernetzung der Parteien mit unterschiedlichen gesellschaftlichen Gruppen der gesellschaftlichen Anbindung der Parteien und damit einer Institutionalisierung der Parteien in einem weiteren Sinne entgegen steht. ${ }^{50}$ Die Auswirkungen der Autonomie südkoreanischer Parteien gegenüber gesellschaftlichen Gruppen sind mithin zumindest als ambivalent einzustufen.

\subsection{Kohärenz}

Spätestens seit der großen Vergleichsstudie von Janda ${ }^{51}$ gilt das Ausmaß an innerparteilichem Faktionalismus als zentraler Indikator für die Kohärenz politischer Parteien. Die Prämisse hierbei ist, dass die Existenz und der Wettbewerb innerparteilicher Faktionen Konsens und Koordination in Parteien sowie allgemein deren Zusammenhalt erschweren. Allerdings hat die vergleichende Faktionalismusforschung gezeigt, dass derartige innerparteiliche Gruppen prinzipiell sowohl dysfunktional als auch funktional für die betroffenen Parteien wie auch das gesamte Parteiensystem sein können. Dysfunktional sind sie z.B. dann, wenn sie die Geschlossenheit, das gemeinsame Vorgehen und damit letztlich die Effektivität von Parteien unterminieren. Auch kann Faktionalismus dazu führen, dass innerparteiliche Personalentscheidungen nicht auf Basis von Verdienst und inhaltlicher Befähigung, sondern auf Basis von Faktionszugehörigkeit getroffen werden. Faktionalismus kann offene Aussprachen be- oder gar verhindern, und inhaltliche Debatten können in den Sog interfaktionaler Machtkämpfe geraten. Andererseits (bzw. in anderen Fällen) können Faktionen aber auch als Transmissionsriemen für Aushandlungsprozesse, Konfliktlösungen und Konsensfindung in Parteien fungieren. Faktionalismus kann gerade in größeren oder dominanten Parteien für den notwendigen innerparteilichen Wettbewerb inhaltlicher und personeller Art sorgen. Die Existenz unterschiedlich ausgerichteter Faktio-

Die zivilgesellschaftlichen Organisationen in der Zeit der ,Regierung des Volkes' von Kim Daejung 1998-2000 - Eine Zwischenbilanz der Aktivitäten und Aufgaben, in: Patrick Köllner (Hrsg.), Korea 2000 - Politik, Wirtschaft, Gesellschaft, Hamburg, 2000, 83-101 (92-96) und Bong-Ki Kim, Die Rolle der Civil Society für die Konsolidierung der Demokratie in Südkorea, in: Patrick Köllner (Hrsg.), Sozialwissenschaftliche Koreaforschung in Deutschland, Hamburg, 2002, 47-75 (64-67).

Siehe hierzu im Einzelnen Köllner (2002, Fn. 8). In der Tat wird in der Literatur zur Institutionalisierung von Parteiensystemen die gesellschaftliche Verankerung von Parteien als ein zentrales Kriterium betrachtet (siehe stellvertretend Mainwaring 1999, Fn. 4, 26).

Janda, (1980, Fn. 17), siehe insb. S. 154. 
nen in einer Partei kann zudem moderierend wirken, indem radikalen oder extremen Positionen bereits in der Partei selbst entgegengewirkt wird. Positiv-integrativ wirkende Faktionen in politischen Parteien können insgesamt auch das Parteiensystem per se stabilisieren. $^{52}$

Auch die Annahme, dass Faktionen zwangsläufig anomischer Natur sind und so zu einer Fragmentierung der Partei beitragen, muss hinterfragt werden. So lassen sich durchaus innerparteiliche Gruppen finden, deren Institutionalisierungsgrad über dem ihrer "Mutterparteien" liegt. Vieles hängt mithin von der spezifischen Art des jeweils vorherrschenden Faktionalismus und der betreffenden Faktionen ab. In Bezug auf den südkoreanischen Fall besteht bei Bebachtern große Einigkeit darüber, dass es sich bei den dortigen innerparteilichen Gruppen um personalisierte Faktionen handelt, die an einzelne Führungspersonen und deren enge Gefolgsleute gebunden sind. Nach Beller und Belloni ${ }^{53}$ weisen personalisierte Faktionen einen klientelistischen Charakter auf; sie sind mithin durch tendenziell asymmetrische Macht- und Ressourcenaustauschbeziehungen zwischen einem Patron und seinen Klienten/Gefolgsleuten gekennzeichnet. Hierarchie und Kommandoketten in personalisierten Faktionen sind zumeist vertikal strukturiert; horizontale Verbindungen zwischen Faktionsmitgliedern werden teilweise sogar unterbunden. Während in der Regel eine gemeinsame Identität der Faktionsmitglieder gegeben ist, bilden klientelistische Verbindungen zum Faktionsführer den zentralen Mobilisierungsmechanismus. Die zeitliche Existenz personalisierter Faktionen reicht üblicherweise nicht über das aktive politische Leben des jeweiligen Faktionsführers hinaus.

Betrachtet man die Literatur zur Entwicklung und Organisation südkoreanischer Parteien genauer, lassen sich hinsichtlich des Faktionalismus zentrale Befunde herausfiltern:

1. Faktionalismus ist ein ubiquitäres Phänomen in der südkoreanischen Parteipolitik. So ist die Geschichte der wichtigeren politischen Parteien des Landes auch immer die Geschichte der Machtkämpfe entsprechender innerparteilicher Gruppen gewesen. Dabei ist es egal, ob man sich die Geschichte der beiden wesentlichen Parteien der Ära Syngman Rhee, der Liberal Party und der Democratic Party, ${ }^{54}$ der Democratic Party

Für eine Diskussion der Arten, Funktionen und Konsequenzen des Faktionalismus in politischen Parteien siehe ausführlicher Patrick Köllner, Faktionalismus in politischen Parteien: Der japanische Fall aus konzeptioneller und komparativer Perspektive, in: Wolfgang Seifert / Claudia Weber (Hrsg.), Japan im Vergleich, München, 2002, 281-306 und die dort angegebene Literatur.

Dennis C. Beller / Frank P. Belloni, Party and Faction: Modes of Political Competition, in: Frank P. Belloni / Dennis C. Beller (Hrsg.), Faction Politics: Political Parties and Factionalism in Comparative Perspective, Santa Barbara / Oxford, 1978, 417-450. 
während der kurzlebigen 2. Republik ${ }^{55}$ oder der bereits erwähnten Democratic Republican Party und der 1967 gegründeten oppositionellen New Democratic Party in der Ära Park Chung-hee ${ }^{56}$ ansieht. Auch in den folgenden Jahrzehnten mangelte es nicht an faktionalen Konflikten, die in zumindest drei Fällen - der New Korea Democratic Party (gegründet 1985), der Democratic Liberal Party (gegründet 1990) und der Democratic Party (gegründet 1991) - aus der Verschmelzung verschiedener Parteien zu einer neuen Partei resultierten. In den drei Fällen wurden im Übrigen nach der jeweiligen Fusion wichtige Parteiämter nach Faktionsproporz vergeben und auch bei früheren Oppositionsparteien scheint sich in einigen Fällen die Stärke der verschiedenen Faktionen in deren Organisations- und Machtstrukturen widergespiegelt zu haben. ${ }^{57}$

2. Vielleicht mit Ausnahme der extrem autoritären Phase Park Chung-hees in den 70er Jahren ist es offenbar über keinen längeren Zeitraum einem Parteiführer gelungen, dem personalisierten Faktionalismus wirkungsvoll entgegenzutreten. ${ }^{58}$ Allerdings scheint die Zentripetalkraft des Machtbesitzes den Faktionalismus in südkoreanischen Regierungsparteien zumindest zeitweise einzudämmen. ${ }^{59}$

3. Während ein gemeinsamer Hintergrund der Mitglieder (Verwandtschaft, Freundschaft, Schule, Region) oder das persönliche Charisma eines Faktionsführers wichtige Beweggründe für den Eintritt in eine bestimmte Faktion darstellen können, sind daneben vor allem persönliche Nutzenkalküle für die Zugehörigkeit bzw. den Wechsel zu einer bestimmten Faktion bestimmend. $^{60}$

4. Lassen sich insbesondere für die 50er und 60er Jahre einige Beispiele finden, in denen inhaltliche, teilweise sogar ideologische Fragen von Relevanz für interfaktionale Kon-

Sung M. Pae, Testing Democratic Theories in Korea, Lanham u.a., 1986, 164-166 und Wonkyoo Lee (1995, Fn. 39), 175-176.

60 Siehe Min (1980, Fn. 54), Kapitel 5; Pae (1986, Fn. 54), 166-167.

Zum Faktionalismus in der DRP und der NDP siehe $Y$. C. Han, Political Parties and Elections in South Korea, in: Se-Jin Kim / Chang-Hyun Cho (Hrsg.), Government and Politics in Korea, Silver Springs, Maryland, 1972, 127-147; Chulsu Kim (1973, Fn. 39), 113-150; Min (1980, Fn. 54), Kapitel 6; Pae (1986, Fn. 54), 167-172, Yong-Ho Kim, Authoritarian Leadership and Party Dynamics: The Rise and Fall of the Democratic Republican Party in South Korea, 1962-1980, unveröffentlichte Dissertation, University of Pennsylvania, 1989, passim und Wonkyoo Lee (1995, Fn. 39), 176-178.

Vgl. Chulsu Kim (1973, Fn. 39), 171; Wonkyoo Lee (1995, Fn. 39), 130-132, 182-185.

$\mathrm{Zu}$ Park Chung-hees ablehnenden Haltung gegenüber innerparteilichen Faktionen und verschiedenen diesbezüglichen Sanktionen in der DRP siehe etwa Bae Ho Hahn, Factions and the Structure of Political Competition in Contemporary Korea: Some Preliminary Observations, Journal of East and West Studies 1 (1971), 59-75 (72-74), Chulsu Kim (1973, Fn. 39), 172-173 und Wonkyoo Lee (1995, Fn. 39), 103-104.

Siehe jedoch Punkt 5.

Vgl. Y. C. Han, (1969, Fn. 56), 456; Hahn (1971, Fn. 58), 66; Wonkyoo Lee (1995, Fn. 39), $185-$ 186. 
flikte waren, steht seither vor allem der Wettbewerb um Macht und Posten im Mittelpunkt der Auseinandersetzungen zwischen verschiedenen innerparteilichen Gruppen.

5. Besonders virulent werden interfaktionale Konflikte oftmals im Jahr der Präsidentschaftswahlen, wenn es darum geht, welche Politiker aus einer Partei für das höchste Amt kandidieren sollen. Dies gilt insbesondere für die Regierungspartei, da nach der Verfassung von 1987 der amtierende Staatspräsident nicht wieder antreten kann. Anschauliche Beispiele aus der jüngeren Zeit bieten die entsprechenden Konflikte in der Democratic Liberal Party 1992, der Grand National Party 1997 und der Millennium Democratic Party 2002. Vor Präsidentschaftswahlen ist auch die Gefahr der Abspaltung einer Faktion am größten, da Spitzenpolitiker, die sich im innerparteilichen Wettbewerb nicht durchsetzen konnten, zuweilen versuchen, auf anderem Wege doch noch für das höchste Amt im Staat zu kandidieren.

Hinsichtlich der spezifischen Form innerparteilicher Faktionen in Südkorea drängt sich der Vergleich mit dem benachbarten Japan auf. Dort haben Faktionen ebenfalls die Parteien des Landes stark geprägt, weisen allerdings insbesondere in der Liberal Democratic Party (LDP) einen deutlich stärker institutionalisierten Charakter auf, sodass man hier von einer Institutionalisierung informeller Parteistrukturen sprechen kann. ${ }^{61}$ Im Gegensatz zu den LDP-Faktionen sind die personalisierten Faktionen in südkoreanischen Parteien deutlich fluider ${ }^{62}$ und simpler strukturiert, sind durch stärkere Machtasymmetrien gekennzeichnet und weisen auch keine institutionalisierten informellen Regeln etwa in Bezug auf die Vergabe von Spitzenämtern in der Partei und der Regierung auf.

Verantwortlich für die unterschiedliche Ausprägung innerparteilicher Faktionen in den beiden ostasiatischen Ländern dürfte neben der langen Regierungszeit der LDP, die eine stärkere Institutionalisierung der Faktionen einschließlich der Entwicklung informeller Regeln überhaupt erst ermöglichte, in Südkorea die mit der langen autoritären Prägung der politischen Kultur verbundene stärkere Konzentration von Macht bei Einzelpersonen und die stärkere Zentralisierung von Spendenflüssen an politische Führer gewesen sein. ${ }^{63}$ Das Wahlsystem hingegen, dessen spezielle Spielart im japanischen Fall für die Entwicklung und starke Institutionalisierung der innerparteilichen Faktionen mitverantwortlich gemacht worden ist, ${ }^{64}$ spielte offenbar im südkoreanischen Fall keine wesentliche Rolle, da hier im

61

62

63

64
Vgl. Köllner (2002, Fn. 52).

Nach Angaben südkoreanischer Politikwissenschaftler sind die beiden einzigen Faktionen, die seit den 80er Jahren zusammengehalten haben, diejenigen von Kim Dae-jung und Kim Young-sam (Interviews, Mai 1999).

Siehe hierzu Wonkyoo Lee (1995, Fn. 39), 188-189.

In Japan existierte bis 1994 das System der nichtübertragbaren Stimmabgabe in mittelgroßen Wahlkreisen, was dazu führte, dass in einem Wahlkreis auch Kandidaten ein- und derselben Partei gegeneinander antraten, vgl. Köllner (2002, Fn. 52). 
Laufe der letzten mehr als 40 Jahre vor allem verschiedene Varianten des Mehrheitswahlsystems in Einerwahlkreisen zum Einsatz gekommen sind. ${ }^{65}$

Interessant sind auch die unterschiedlichen Konsequenzen des Faktionalismus in japanischen und südkoreanischen Parteien. Während dem Faktionalismus in japanischen Parteien ein gehöriges $\mathrm{Ma} ß$ an negativen Auswirkungen attestiert wird - Schwächung der Parteiführungen, Vergabe von Posten nach Seniorität statt nach Verdienst, Anfachen des Wettbewerbs um politische Spenden etc. - sind andererseits auch positive Effekte - Gewährleistung von Meinungspluralismus und verschiedenen personellen Optionen in der LDP, Verhinderung einer diktatorischen Herrschaft über die Partei, Stabilität durch institutionalisierte Machtverteilungsregeln etc. - auszumachen. Demgegenüber ist dem Faktionalismus in südkoreanischen Parteien durchweg bescheinigt worden, die Stabilität der Parteien unterminiert und insgesamt die demokratische Entwicklung gehindert zu haben.

\section{Ursachen der spezifischen Entwicklung südkoreanischer Parteien}

Wie die vorangegangene Anwendung der Kriterien der Anpassungsfähigkeit, der Komplexität, der Autonomie und der Kohärenz auf südkoreanische Parteien gezeigt haben sollte, sind diese insgesamt durch eine nur schwach ausgeprägte Institutionalisierung gekennzeichnet. Worauf lässt sich dies zurückführen? Ragsdale und Theis ${ }^{66}$ haben in einem anderen Kontext festgehalten, dass Institutionalisierung - ob nun starker oder schwacher Art aus dem Zusammenspiel der individuellen Interessen in einer Organisation und Aspekten der Umwelt der jeweiligen Organisation resultiert. $\mathrm{Zu}$ den systemischen Aspekten der Umwelt, die die Charakteristika politischer Parteien beeinflussen, können länderspezifische sozioökonomische und politische Faktoren gezählt werden, wobei hinsichtlich der politischen Faktoren das besondere Augenmerk dem konstitutionellen Umfeld gelten muss, in dem sich die Parteien bewegen. ${ }^{67}$ Ferner müssen die spezifischen historischen Rahmenbedingungen berücksichtigt werden, innerhalb derer die Parteien sich entwickeln. Im Folgenden werden wesentliche historische, institutionelle und attitudinale Faktoren aufgezeigt, die für das Verständnis der spezifischen Entwicklung südkoreanischer Parteien maßgeblich sind.

65

66

67

Vgl. Croissant (2001, Fn. 26); Mo / Brady (2001, Fn. 42).

Lyn Ragsdale / John J. Theis, III, The Institutionalization of the American Presidency, 1924-92, American Journal of Political Science 41 (1997), 1280-1318 (1283).

Vgl. Robert Harmel / Kenneth Janda, Parties and Their Environment. Limits to Reform?, New York / London, 1982, 12-13. 


\subsection{Historische Ausgangslage und Aspekte der politischen Kultur ${ }^{68}$}

Bereits die Ausgangsbedingungen für die Entwicklung voll ausgebildeter "multifunktionaler" Parteien des liberaldemokratischen Idealtypus waren in Südkorea nicht eben günstig. Zunächst förderten die Wirren nach der Befreiung und die Fragmentierung der nationalistischen Bewegung, die während des japanischen Kolonialregimes (1910-1945) gewachsen war, die Entstehung einer extrem zersplitterten Parteienlandschaft. ${ }^{69}$ So ließen sich im Juni 1946107 Parteien bei der amerikanischen Militärregierung registrieren, ein Jahr später war ihre Zahl auf 344 angewachsen. ${ }^{70}$

Die meisten dieser so genannten Parteien waren reine Faktionen (hier im Sinne von Protoparteien), die von einzelnen Politikern mit spezifischen, oftmals lokal begrenzten Anliegen gebildet wurden. Bis in die späten 50er Jahre blieb die Anzahl unabhängiger Abgeordneter in der Nationalversammlung, dem südkoreanischen Parlament, recht hoch. ${ }^{71}$ Viele Koreaner wählten "ihren" Abgeordneten aufgrund von regionalen, schulischen oder verwandtschaftlichen Bindungen; Bindungen an Parteien auf Basis programmatischer oder ideologischer Unterstützung existierten so gut wie nicht. Sie entsprachen auch nicht dem Repräsentationsverständnis der meisten Koreaner, die in ihrem Abgeordneten eher einen Delegierten sahen, der sich für lokale Belange einzusetzen hatte, denn einen Treuhänder, der sich auf nationaler Ebene um allgemeine politische Belange kümmern sollte. ${ }^{72}$ Eine mögliche ideologisch-programmatische Differenzierung der politischen Parteien Südkoreas wurde nicht zuletzt dadurch verhindert, dass sich im Gefolge der Teilung der koreanischen Halbinsel kommunistische oder auch nur "progressiv" orientierte Parteien nicht entfalten konnten. Anti-Kommunismus wurde als Mittel zur politischen Manipulation und Kontrolle eingesetzt. Dies verstärkte die inhärente Tendenz zur Reduzierung der zunehmend konservativeren Parteipolitik auf Machtkämpfe persönlicher Natur. ${ }^{73}$

68

69

70

71

72

73

Dieser Unterabschnitt stützt sich auf Köllner (2002, Fn. 8), 3-5.

Siehe dazu Joung-Sik Lee, Development of Party Politics in Korea, Koreana Quarterly 9 (1967), $1-17(7-9)$

Chulsu Kim (1973, Fn. 39), 40. Zur Entwicklung der wichtigsten Parteien von der unmittelbaren Nachkriegszeit bis Ende der 50er Jahre siehe übersichtsartig Tae-Soo Han (1962, Fn. 54) und Joung-Sik Lee (1967, Fn. 69), 7-14.

Siehe hierzu Soong Hoom Kil / Chung-in Moon (Hrsg.), Understanding Korean Politics. An Introduction, Albany, NY, 2001, 44-45, Tabelle 3.2.

Vgl. Chi-Young Pak, Political Opposition in Korea, 1945-1960, Seoul, 1980, 19-20; Chong Lim Kim / Joel D. Barkan / Ilter Turan / Malcolm E. Jewell, The Legislative Connection: The Politics of Representation in Kenya, Korea, and Turkey, Durham, North Carolina, 1984, 89.

Vgl. Chulsu Kim (1973, Fn. 39), 294-295; Soong Hoom Kil, Development of Korean Politics - A Historical Profile, in: Kil / Moon (Hrsg.), a.a.O. (Fn. 71), 33-69, 39-42; Sung Chul Yang, The North and South Korean Political Systems. A Comparative Analysis, Revised Edition, Seoul, 1999, 192-201. 
Zudem kann auch nicht übersehen werden, dass das wiederholte Versagen konstitutioneller Machtausübung in Südkorea, das seinen Ausdruck von den späten 40er bis in die 80er Jahre in Regierungsumstürzen, Militärcoups, der Verhängung von Kriegsrecht und zahlreichen machtpolitisch motivierten Verfassungsänderungen gefunden hat, ${ }^{74}$ nicht spurlos an der Entwicklung der politischen Parteien vorbeigegangen ist. Vom zeitweisen Verbot politischer Parteien zu Anfang der 60er Jahre und 1980 einmal ganz abgesehen, stellten die wiederholten konstitutionellen Krisen auch immer wieder die Funktionsfähigkeit der politischen Parteien und des Parteiensystems insgesamt in Frage. Dabei erscheint es eher zweitrangig, ob die Schwäche der Parteien das Versagen konstitutioneller Herrschaft bedingte oder umgekehrt. ${ }^{75}$ Tatsache bleibt in jedem Fall, dass es bis zur demokratischen Transition Ende der 80er Jahre keinen friedlichen Machtwechsel auf der Basis veränderter Mehrheitsverhältnisse der Parteien im Parlament gab.

Schließlich lässt sich argumentieren, dass auch die Einbettung der Parteien in die vorherrschende politische Kultur des Landes deren Gestalt zumindest beeinflusst hat. Erwähnt werden müssen in diesem Zusammenhang weiterhin verbreitete autoritäre Einstellungen sowie eine auffällige Orthodoxie im Denken und Handeln, die sich auch auf die starke neokonfuzianische Prägung des Landes in den letzten Jahrhunderten zurückführen lassen. Insgesamt lässt sich eine nicht nur formal-institutionell (siehe dazu unten), sondern auch kulturell unterfütterte Tendenz zur Zentralisierung und Konzentration von Macht in Korea ausmachen. Darüber hinaus kann argumentiert werden, dass die Orientierung der Koreaner an Primärgruppen und der entsprechende Mangel an unversalisiertem Vertrauen keine gute Grundlage für eine Institutionalisierung politischer Parteien geboten hat. ${ }^{76}$

\subsection{Die institutionelle Konfiguration des Regierungssystems}

Wie in zahlreichen akteur- und institutionell orientierten Theorien argumentiert worden ist, stellt die formale institutionelle Konfiguration eines politischen Systems eine "Gelegenheitsstruktur" dar, die über spezifische Anreize und Sanktionen auch das Verhalten und die Struktur von Parteien beeinflusst. ${ }^{77}$ In Bezug auf die Konfiguration von Regierungssyste-

Siehe hierzu überblicksartig Kil (2001, Fn. 73), 36-37, Tabelle 3.1, und John Kie-Chang Oh, Korean Politics. The Quest for Democratization and Economic Development, Ithaca / London, 1999, 102-103.

75

Für eine Argumentation, die die erstgenannte Kausalwirkung betont, siehe Chulsu Kim (1973, Fn. 39).

Siehe hierzu Wonkyoo Lee (1995, 22), Kapitel 3-5, Köllner (1999, Fn. 41), 58-61, und Woon-Tai Kim, Korean Politics: Setting and Political Culture, in: Kil / Moon (Hrsg.), a.a.O., 9-32 (19-21, 24-29).

Wichtig ist in diesem Zusammenhang der Zusatz "formal", da die institutionelle Konfiguration von politischen Systemen auch informelle Elemente - Stichworte: Klientelismus, Faktionalismus aufweist (siehe dazu Joachim Betz / Patrick Köllner, Informale Politik im internationalen Ver- 
men hat Samuels in diesem Kontext jüngst aufgezeigt, dass Präsidential- und semipräsidentielle Systeme die Entwicklung von "präsidentialisierten Parteien" befördern. Diese sind durch a) einen Fokus auf spezifische Kandidaten (statt etwa Programme) bei Wahlen, b) eine Ausrichtung auf Stimmenmaximierung (statt die Erringung von Regierungsämtern oder die Durchsetzung spezifischer inhaltlicher Anliegen), c) eine nachrangige Bedeutungszumessung in Bezug auf Parlamentswahlen und d) eine beschränkte Bedeutung der Parteiorganisation gekennzeichnet. ${ }^{78}$ Hier soll nun argumentiert werden, dass Südkoreas Regierungssystem, das durch eine starke Zentralisierung und eine deutliche Machtkonzentration beim Staatspräsidenten charakterisiert war und ist, wirkungsmächtige Anreize geliefert hat, die einer starken Institutionalisierung der politischen Parteien des Landes eher entgegen wirkten. Dies wird im Folgenden kurz ausgeführt.

Die erste Verfassung der 1948 gegründeten Republik Korea sah für das Land ein demokratisches politisches System vor, das Elemente des britischen Parlamentarismusmodells mit Elementen des amerikanischen Präsidentialmodells verband. Während die Verfassungsbestimmungen zu diesem semipräsidentiellen System auf den ersten Blick der Legislative eine starke Rolle zusprachen, existierten bei näherem Hinsehen zahlreiche Möglichkeiten, aus denen der Staatspräsident machtvolle Befugnisse nicht nur im exekutiven Bereich schöpfen konnte. So sah die Verfassung - um nur ein Beispiel zu nennen - die Möglichkeit von Präsidentialerlassen vor. Ob und wie diese Spielräume von den Staatspräsidenten des Landes genutzt würden, hing nicht zuletzt vom Rollenverständnis des ersten Amtsinhabers ab. Mit Syngman Rhee wurde ein Mann zum Staatspräsidenten gewählt, der trotz seiner langen Jahre in den USA demokratischen Prinzipien nur oberflächlich verbunden war. Das Rollenverständnis von Rhee, der selbst einer Aristokratenfamilie entstammte, wurzelte offenbar eher in den elitären Einstellungen der yangban-Aristokratie während der koreanischen YiDynastie (1392-1910). Rhee, der während seiner dreizehnjährigen Amtszeit zunehmend autoritärere Züge offenbarte, scheute nicht einmal davor zurück, von ihm gewünschte Verfassungsänderungen durch Manipulationen oder Androhungen physischer Gewalt zu erwirken. ${ }^{79}$ In den letzten Jahren seiner Amtszeit ähnelten Rhee und seine willfährigen Minister "an old dynastic court of a king and his yangban officials". ${ }^{80}$ Es überrascht daher nicht,

gleich: Ein Forschungsaufriss, Nord-Süd aktuell 15 (2001), 94-104; Croissant (2001, Fn. 8), 4652. An dieser Stelle soll uns jedoch nur das formale, d.h. in Verfassungen, Gesetzen etc. schriftlich fixierte institutionelle Gefüge interessieren. Auf eine Diskussion der parteibezogenen Auswirkungen der formalen Institution des Wahlsystems soll hier verzichtet werden, da Südkoreas oftmals verändertes Wahlsystem für das Parlament eher das Parteiensystem und weniger die Parteien per se beeinflusst hat; siehe dazu Croissant (2001, Fn. 26), 70-90; Chan Wook Park 2002, Fn. 44), 127-133.

David J. Samuels, Presidentialized Parties. The Separation of Powers and Party Organization and Behavior, Comparative Political Studies 35 (2002), 461-483 (478).

Vgl. John Oh (1999, Fn. 74), 25-40.

80

Oh, (1999, Fn. 74), 40. 
dass Rhee für die politischen Parteien des Landes nur wenig übrig hatte (siehe dazu auch Abschnitt 4.3).

Nicht nur Rhees spezifisches Rollenverständnis sollte für die autoritären Regime der 60er bis 80er Jahre prägend wirken; auch die von ihm eingeführte "Tradition" von Verfassungsänderungen mit dem Ziel des Erhalts und der Sicherung der politischen Macht wurde von den ehemaligen Generälen im Staatspräsidentenamt, Park Chung-hee und Chun Doo-hwan (1980-1987), weiter gepflegt. ${ }^{81}$ In der Folge entwickelte sich das höchste Staatsamt Südkoreas zur viel beklagten "imperialen Präsidentschaft". Im Vergleich zu ihr kam anderen politischen Organen wie der Nationalversammlung oder eben den Parteien nur eine marginale Rolle im politischen Entscheidungsprozess zu - was im Übrigen deren Institutionalisierung auch nicht eben erleichterte. ${ }^{82}$ Insgesamt kann dem Urteil von Oh über das politische Systems Südkoreas bis zur demokratischen Öffnung 1997 zugestimmt werden: "[D]emocracy was only an institutional framework within which strong rulers exercised almost unlimited power". 83

Zwar wurden im Rahmen der letzten Verfassungsreform 1987 die Machtbefugnisse des Staatspräsidenten wieder beschnitten, doch im internationalen Vergleich verfügt über er dank des Vorsitzes des Staatsrats (Kabinetts), der Ernennung des Ministerpräsidenten sowie anderer exekutiver und legislativer Möglichkeiten einerseits und einer beschränkten Anzahl von politischen "Vetospielern" andererseits über eine vergleichsweise machtvolle Stellung. ${ }^{84}$ Die zentrale Position des Staatspräsidenten wird zudem gestützt durch seinen Zugriff auf den gut entwickelten Verwaltungsapparat und die starke politische Zentralisierung Südkoreas, an der auch die zögerliche Ausweitung lokaler Autonomie in den 90er Jahren nur wenig in substantieller Hinsicht geändert hat.

Für ambitionierte Politiker bildet in Südkorea der Einzug in das "Blaue Haus", den Sitz des Staatspräsidenten, weiterhin das höchste Ziel. Bekanntlich befördern schwache und wenig einflussreiche Parteien vor allem die Macht der Exekutive. Vor diesem Hintergrund bestand und bestehen aus Sicht dieser politischen Führer Südkoreas nur wenig Anreize, die Institutionalisierung der politischen Parteien voranzutreiben. Allgemein betrachtet dienten

Für eine Diskussion der Verfassungsänderungen bis 1980 und der damit einhergehenden Machtbefugnisse des Präsidenten siehe Pae (1986, Fn. 54), Kapitel 7.

Zum Machtverhältnis zwischen Präsident und Parlament seit 1948 siehe auch Seong-Ho Lim, A Paradox of Korean Democracy. 50 Years' Experience of the 'Imperial' Presidency and the 'Peripheral' Legislature, Korea and World Affairs 22 (1998), 522-538.

Kongdan Oh, The Korean War and South Korean Politics, in: David McCann / Barry S. Strauss (Hrsg.), War and Democracy. A Comparative Study of the Korean and the Peleponnesian War, Armonk / London, 2001, 176-187 (185). 
und dienen politischen Parteien vor allem als Instrumente zur Verfolgung persönlicher Interessen. Diese resultierte, um mit Kang ${ }^{85} \mathrm{zu}$ sprechen, "in politicized rather than institutionalized parties". Dieser streng utilitaristische Ansatz gegenüber politischen Parteien fand seinen Widerhall auch in der Haltung wichtiger politischer des Landes gegenüber den Parteien, auf die im folgenden Abschnitt eingegangen werden soll.

\subsection{Die Haltung politischer Führer Südkoreas gegenüber den Parteien}

Was neben den bisher erwähnten historischen Rahmenbedingungen und Aspekten der politischen Kultur sowie dem formalen institutionellen Gefüge des Regierungssystems einer Institutionalisierung der südkoreanischen Parteien schließlich auch entgegenstand, war der Widerstand der politischen Führer des Landes, eigenständige Parteien mit innerparteilicher Demokratie oder auch nur einer Dezentralisierung von Entscheidungskompetenzen zu akzeptieren. So machten beispielsweise die beiden langjährigen Staatspräsidenten Syngman Rhee und Park Chung-hee keinen Hehl aus ihrer Abneigung gegenüber politischen Parteien, die sie lediglich als notwendiges Vehikel zur Legitimierung und Sicherung ihrer persönlichen Macht betrachteten. ${ }^{86}$ Sung-joo $\mathrm{Han}^{87}$ hat die Haltung Parks gegenüber den politischen Parteien des Landes und deren Konsequenzen wie folgt zusammengefasst:

„He was suspicious of both the progovernment and opposition parties, and considered them necessary evils at best, a threat at worst. Neither this own Democratic Republican Party nor the Opposition New Democratic Party was given an opportunity to develop a leadership structure or cultivate grassroots support. As a result, neither party could attract high-caliber people or induce strong identification on the part of a large number of citizens. Thus, political parties failed to become the principal vehicle through which the struggle for power and the opportunity to make policy could be carried out following Park's death.“

Nicht zuletzt am Widerstand Park Chung-hees scheiterten denn auch Pläne in den frühen 60er Jahren, die Regierungspartei Democratic Republican Party (DRP) zu einer "modernen" Partei mit klarer ideologischer Ausrichtung und dezentralisierten Machtstrukturen zu formen. ${ }^{88}$ Trotz einer offiziellen Mitgliederzahl von 1,7 Millionen in den 60er Jahren gelang der DRP - wie auch allen vorherigen und nachfolgenden Parteien - keine umfas-

David C. Kang (2001), The Institutional Foundation of Korean Politics, in: Kil / Moon (Hrsg.), a.a.O. (Fn. 71), 71-105 (88).

Vgl. Ki-Shik Han, Development of Parties and Politics in Korea (II), Korea Journal 14 (1974), 4157 (44-46); Chulsu Kim (1973, Fn. 39), Kapitel 2.

87

Sung-joo Han, Political Institutionalization in South Korea, 1961-1984, in: Robert A. Scalapino / Seizaburo Sato / Jusuf Wanandi (Hrsg.), Asian Political Institutionalization, Berkeley, 1986, 116137 (120).

88

Siehe hierzu Chulsu Kim (1973, Fn. 39), 176-185. 
sende Institutionalisierung. Der schrittweise Machtverlust des Parteisekretariats, die zunehmende Abhängigkeit der Partei von der staatlichen Bürokratie und schließlich die mangelnde Autonomie der DRP gegenüber der personalisierten Führung von Staatspräsident Park führten dazu, dass die Partei unkonsolidiert blieb und konsequenterweise nach dem Tode Parks aufgelöst wurde. ${ }^{89}$

Insgesamt lässt sich festhalten, dass de facto alle Regierungs- und Oppositionsparteien in den vergangenen Jahrzehnten primär der Machterringung und -sicherung einzelner Politiker dienten. Diese sahen die Parteien vor allem als notwendiges Instrument zur Stimmenmobilisierung an und zeigten entsprechend kein Interesse an einer Institutionalisierung, die ihre persönliche Kontrolle und Führerschaft in Frage hätte stellen können. Schwach institutionalisierte Partei in ihrer Form als oftmals regional verankerte Wahlvereine waren und sind für südkoreanische Spitzenpolitiker mithin durchaus funktional. Dass sie wegen ihrer schwachen Institutionalisierung nur im eingeschränkten Maß öffentliche Funktionen wahrnehmen können, die für das gesamte politische System für Bedeutung sind, steht auf einem anderen Blatt.

\section{Zusammenfassung und Fazit}

In diesem Artikel wurde der Versuch unternommen, die Institutionalisierung südkoreanischer Parteien zu analysieren und die Ursachen für deren spezifische Entwicklung zu bestimmen. Mit Hilfe der Huntingtonschen Institutionalisierungskriterien der Anpassungsfähigkeit, der Komplexität, der Autonomie und der Kohärenz ist dabei zunächst gezeigt worden, dass die Parteien Südkoreas durch eine vergleichsweise schwache Institutionalisierung gekennzeichnet sind. So weisen südkoreanische Parteien erstens eine kurze durchschnittliche Lebensdauer und bisher nur beschränkte Wechsel in den Parteiführungen sowie zwischen Oppositions- und Regierungsrollen auf. Zweitens ist eine weitgehend nominale Natur der formalen Parteiorganisation und ein ausgeprägter Personalismus in den Parteien erkennbar. Drittens existieren nur schwache Verbindungen zwischen den Parteien und anderen sozialen Gruppen, was in Bezug auf die gesellschaftliche Anbindung der Parteien durchaus als problematisch angesehen werden kann. Viertens sind südkoreanische Parteien durch personalisierte Faktionen gekennzeichnet, die tendenziell die Stabilität der Parteien unterminieren. Der Vergleich mit japanischen Parteien, insbesondere der langjährigen Regierungspartei LDP, zeigt zudem, dass informelle Elemente der Parteiorganisation (persönliche Unterstützervereinigungen von Politikern auf der lokalen Ebene und innerparteiliche Faktionen auf der zentralen Ebene) in Südkorea deutlich schwächer Institutionalisiert sind als dies im Nachbarland der Fall ist. 
Hinsichtlich der Ursachen der schwachen Institutionalisierung südkoreanischer Parteien wurde zunächst auf historische Rahmenbedingungen und Aspekte der politischen Kultur des Landes verwiesen, die einer Stabilisierung und Routinisierung der Parteien nicht förderlich waren. In Bezug auf die historischen Rahmenbedingungen wurde hierbei abgehoben auf die Fragmentierung der nationalistischen Bewegung und die daraus resultierende Zersplitterung der Parteienlandschaft nach der Unabhängigkeit, die Teilung der Halbinsel und die damit verbundene deutliche Beschränkung ideologischer Differenzierung der Parteien durch den starken Anti-Kommunismus in Südkorea sowie schließlich die wiederholten konstitutionellen Krisen, die eine Kontinuität der Parteientätigkeit verhinderten. Zu den Aspekten der politischen Kultur, die der Institutionalisierung politischen Parteien nicht förderlich waren, können das spezifische Repräsentationsverständnis der südkoreanischen Bevölkerung (Abgeordnete als Treuhänder lokalen Interessen) sowie autoritäre Einstellungen und ein starker Personalismus gezählt werden.

Die starke Stellung des Staatspräsidenten im institutionellen Regierungsgefüge Südkoreas lieferte zudem deutliche Anreize für führende Politiker, persönliche Machtambitionen vor den Aufbau starker Parteien zu stellen. Damit verbunden ist der letzte aufgeführte Faktor, der einer Institutionalisierung politischer Parteien in Südkorea entgegenstanden hat: die Haltung politischer Führer gegenüber den Parteien. In diesem Zusammenhang wurde gezeigt, dass wichtige politische Führer Südkoreas Parteien vor allem als Werkzeug zur Erreichung und Sicherstellung persönlicher Macht betrachtet haben. An dieser Sichtweise hat sich bis heute wenig verändert. Insgesamt entsprechen südkoreanische Parteien dem Typus charismatischer Parteien im Sinne Angelo Panebiancos, bei denen der Parteiführer "has no interest in organizational reinforcement which would inevitably set the stage for the party's "emancipation" from his control". 90

Während es letztlich nahezu unmöglich sein dürfte, genau zu bestimmen, welche der genannten Ursachen der schwachen Institutionalisierung südkoreanischer Parteien am bedeutendsten waren, kann zumindest die These aufgestellt werden, dass die genannten historischen Rahmenbedingungen und Aspekte der politischen Kultur zwar insgesamt eine schwache Institutionalisierung begünstigten, dass aber letztendlich die Reaktion der politischen Führer Südkoreas auf institutionelle Anreize und ihre Haltung gegenüber den Parteien die eigentlich bestimmenden Faktoren in diesem Kontext bildeten. In jedem Fall lässt sich in Konkretisierung der Feststellung von Ragsdale und Theis zu den Ursachen der Institutionalisierung von Organisationen festhalten, dass die schwache Institutionalisierung der südkoreanischen Parteien aus dem Zusammenspiel der Interessen und damit verbundenen Haltungen von Parteiführern einerseits und historischen, gesellschaftlichen und politischen Aspekten der Umwelt der Parteien andererseits resultierte. 
In konzeptioneller Hinsicht hat die Studie zum einen unterstrichen, dass es bei der Beschäftigung mit der Institutionalisierung politischer Parteien (oder anderer Organisationen) sinnvoll ist, die Aspekte der Wertschätzung und der Stabilisierung/Routinisierung voneinander zu trennen. Zwar lässt sich für den südkoreanischen Fall zeigen, dass sowohl eine niedrige Wertschätzung als auch eine niedriger Grad an Stabilisierung/Routinisierung gegeben sind, doch scheint hier die niedrige Wertschätzung auch eine Ursache für die schwache organisatorische Stabilität und verhaltensbezogene Routinisierung darzustellen.

Zum anderen legt die Studie nahe, dass eine differenzierte Auseinandersetzung mit den Institutionalisierungskriterien der Autonomie und der Kohärenz notwendig ist. Der südkoreanische Fall signalisiert, dass - anders als von Huntington angenommen - eine relativ große Autonomie politischer Parteien gleichbedeutend mit Schwächen bei der Institutionalisierung sein kann. Entscheidend ist in diesem Kontext mithin, ob die gesellschaftliche Anbindung der Parteien mit in die Analyse einbezogen wird. Auch gilt es in Bezug auf das Kriterium der Kohärenz, genauer: den Indikator der Faktionalisierung differenziert vorzugehen. Zwar kann die Existenz und der Wettbewerb innerparteilicher Faktionen - wie in früheren Ansätzen postuliert und im südkoreanischen Fall auch gegeben - die Stabilität von politischen Parteien unterminieren. Dass dies jedoch nicht unbedingt der Fall sein muss, legt der Vergleich mit dem japanischen Fall nahe, auf den hier ebenfalls kurz eingegangen wurde. In forschungsstrategischer Hinsicht erscheint es ratsam, in Untersuchungen zur Institutionalisierung politischer Parteien (und anderer Organisationen) nicht nur die Institutionalisierung formaler und informeller Organisationsstrukturen sowie Verhaltensmuster zu unterscheiden, sondern auch die möglicherweise unterschiedlichen Konsequenzen der Institutionalisierung in diesen beiden Bereichen zu berücksichtigen. Damit einhergehend sollte das Augenmerk der Dynamik des Zusammenspiels der Institutionalisierung formaler und informeller Elemente gelten. Ein derartige differenzierte Perspektive erscheint für die Forschung zur Institutionalisierung politischer Parteien von großem heuristischem Wert. 